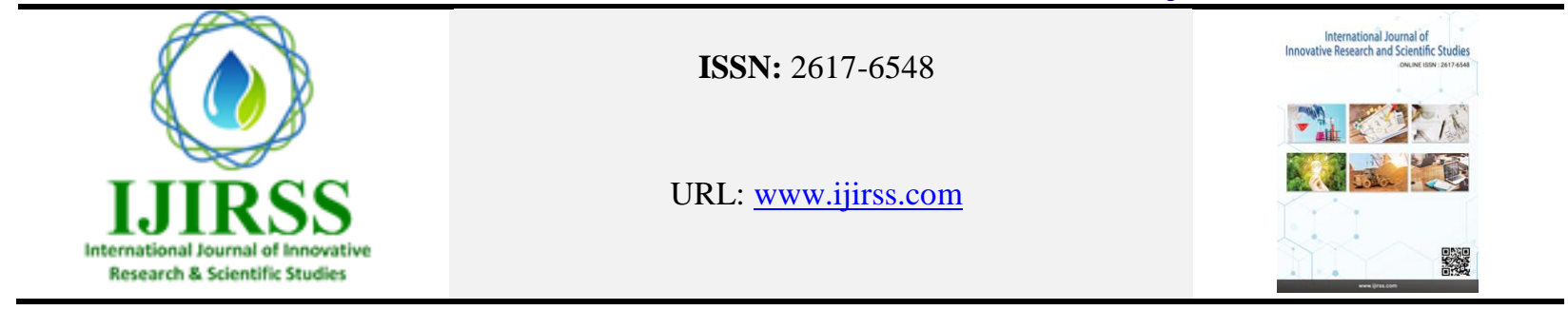

\title{
Studying the Effects of Potassium Phosphite (1\%) on Physiological Changes in Potato (Solanum tubrusum L.) Tuber Under Late Blight Stress
}

\author{
Mohammad Aqa Mohammadi ${ }^{1,2 *}$, Fraidoon Karimi ${ }^{2}$, Mohammad Naser Taheri $^{2}$, Naveedullah Sediqui ${ }^{2}$ \\ ${ }^{1}$ College of Horticulture, Fujian Agriculture and Forestry University, Fuzhou 350002, China \\ ${ }^{2}$ Department of Horticulture, College of Agriculture, Alberoni University, Kohistan 1254, Kapisa, Afghanistan \\ *Corresponding author: Mohammad Aqa Mohammadi (mohammadaqam85@gmail.com)
}

\begin{abstract}
Phosphite-based fungicides are a group of chemicals that are directly harmless to the environment and have a direct fungicide effect, while also indirectly stimulating the plant immune response. Potato late blight is one of the most destructive potatoes diseases globally, causing many morphological and physiological changes in potato leaves and tubers. In current experiment, the potato plants with three-time spray of potassium phosphite (KPhi) on potato leaves, following three months in storage, and inoculated with late blight were investigated. This study examined the possible role of KPhi in delays of late blight invasion and physiological responses including the reactive oxygen species (ROS) metabolism in potato tubers after challenging with late blight pathogen. Samples were collected at 0, 24, 48, 72 and 96 hours of time points for evaluations. Antioxidant enzymes such as superoxidase dismutase (SOD), peroxidase (POD) and catalase (CAT), in addition content of metabolites such as phytoalexin and phenolic after infection with pathogen was investigated. Meanwhile, KPhi treatment resulted in plant tolerance with improved resistance, increased antioxidant enzymes activities and non-enzymatic compounds compare to untreated plants under similar stress. The results of this study indicate that the role of KPhi reduces the adverse effects of pathogens, suppress potato late blight and improves antioxidant enzymes activities and non-enzymatic compounds in potato tubers.
\end{abstract}

Keywords: Antioxidant Enzymes, Non-enzymatic Compounds, Potato, Potassium Phosphite, Late Blight.

DOI: 10.53894 /ijirss.v3i4.41

Funding: This study received no specific financial support.

History: Received: 18 February 2020/Revised: 2 September 2020/Accepted: 25 September 2020/Published: 5 October 2020

Licensed: This work is licensed under a Creative Commons Attribution 4.0 License $(\mathrm{oc})$ EY

Acknowledgement: All authors contributed to the conception and design of the study.

Competing Interests: The authors declare that they have no conflict of interests.

Transparency: The authors confirm that the manuscript is an honest, accurate, and transparent account of the study was reported; that no vital features of the study have been omitted; and that any discrepancies from the study as planned have been explained.

Ethical: This study follows all ethical practices during writing. 


\section{مطالعه تآثيرات يوتاثيم فاسفايت (1\% ) بالاى تغييرات فزيولوزيكى در تيوبركجالو تحت شر ايط تنش بلايت بسني"}

محمدآقا محمدى 2،1، ، فريدون كريمى2، محمدناصر طاهرى2، نويداله صديقى2

1دانشكده هارتبكلجر، دانشخاه زراعت و جنكلدارى فوجيان، فوجو 350002، هِين

2 دبيارتمنت هارتبكلجٍر، دانشكده زراعت، دانشكاه البيرونس، كوهستان 1254، كابيسا، افغانستان

خلاصه

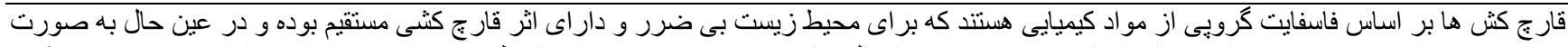

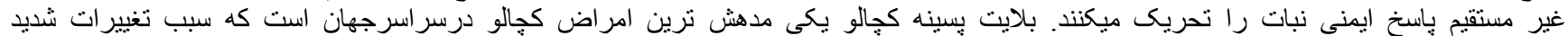

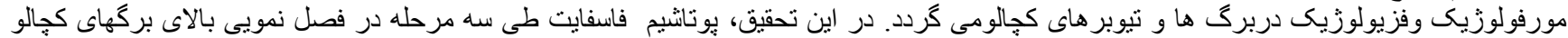

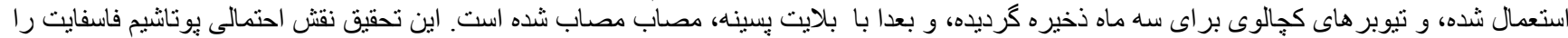

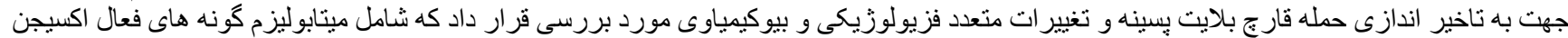
(Reactivity Oxygen Species)

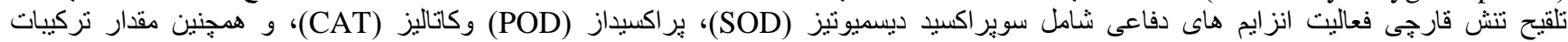

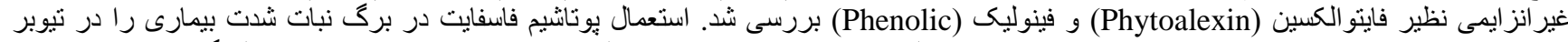

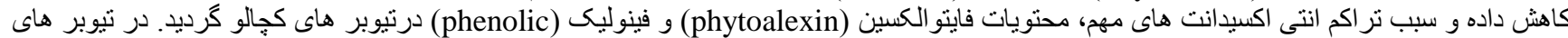

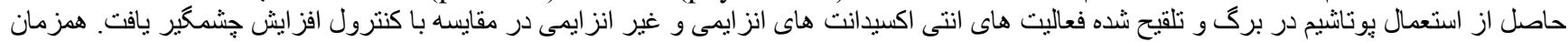

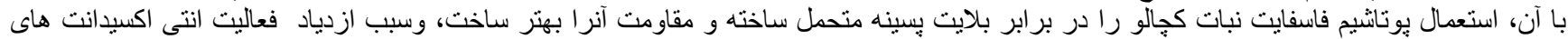

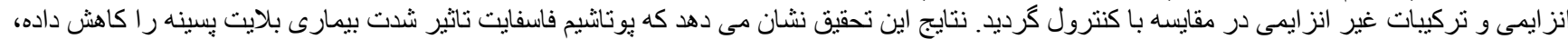

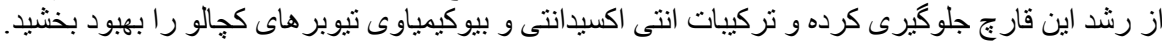

كلمات كلبِى: انتى اكسيدانت هاى انز ايمى، انتى اكسيدانت هاى غير انز ايمى، كجالو، بِوتاثيم فاسفايت، بلايت بِّينه

مقدمه

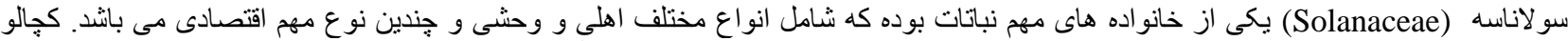
(Solanum tuberosum.)

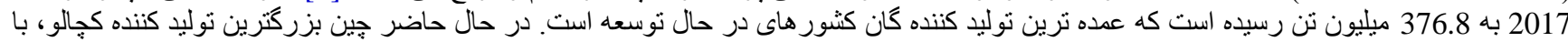

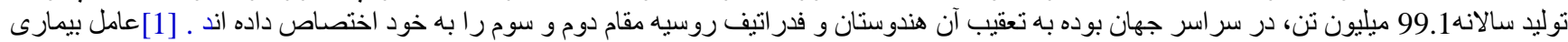

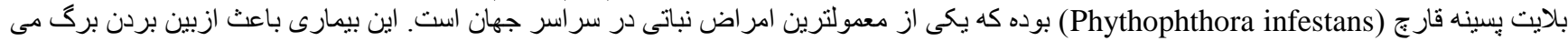

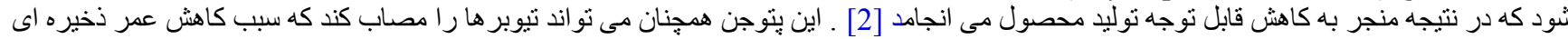

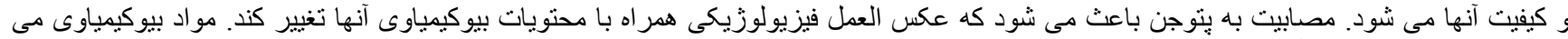

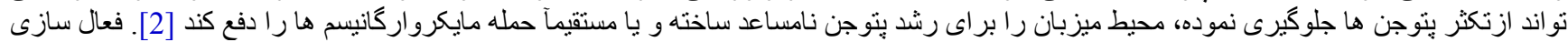

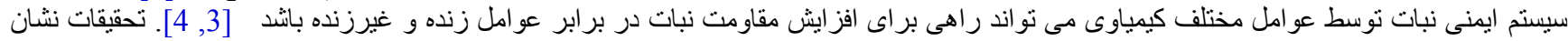

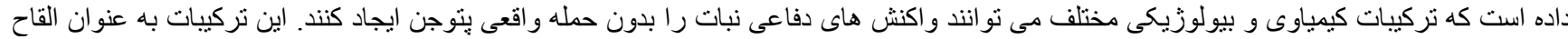

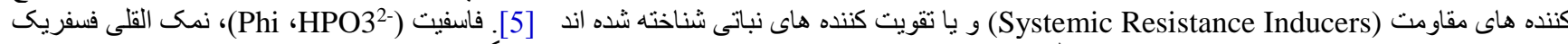

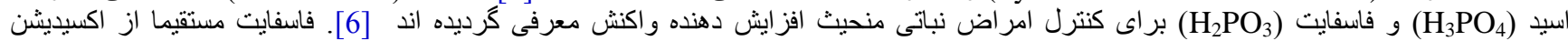

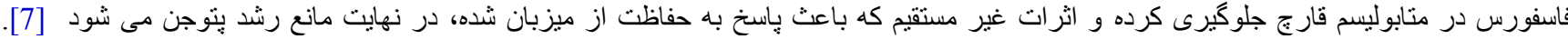

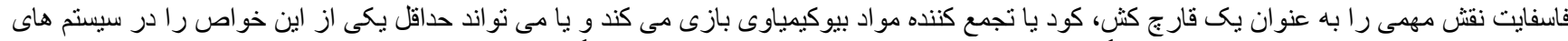

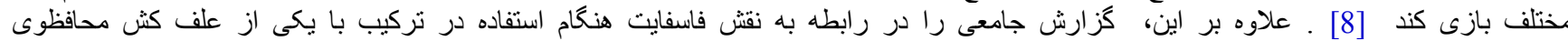
Chlorothalonyl

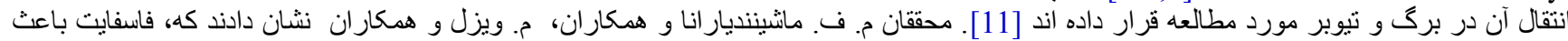

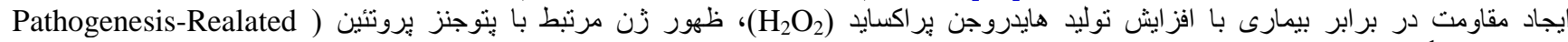

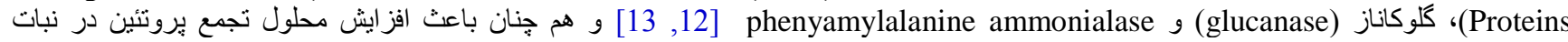

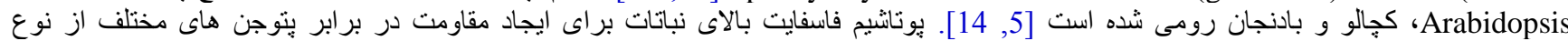

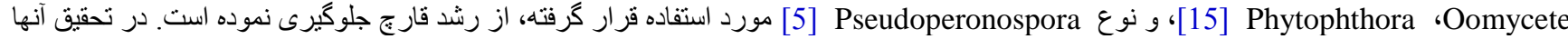

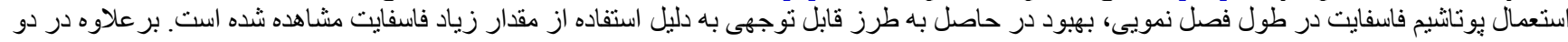

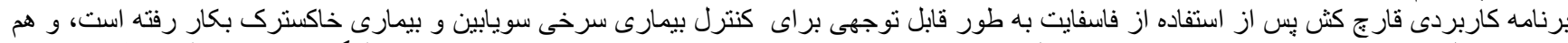

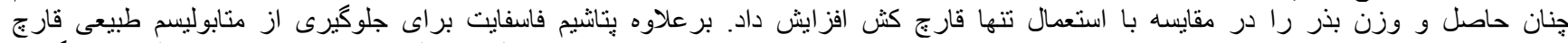
إن مycetes

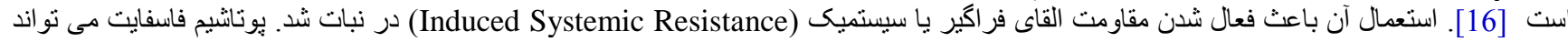

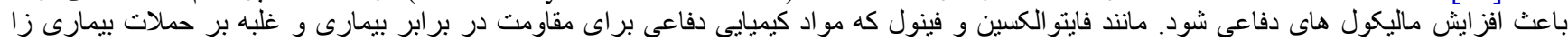

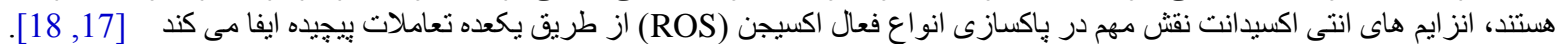

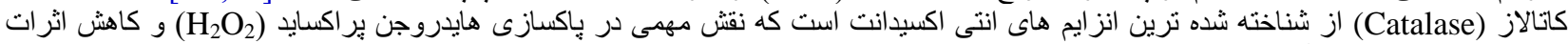

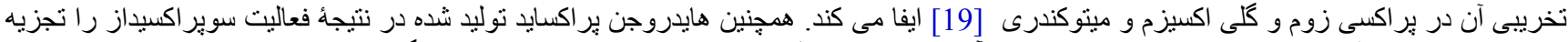

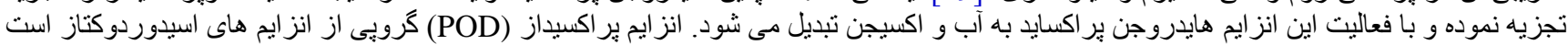




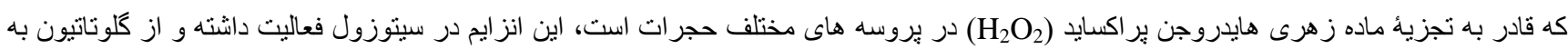

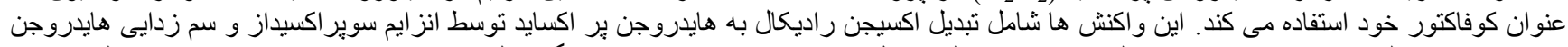

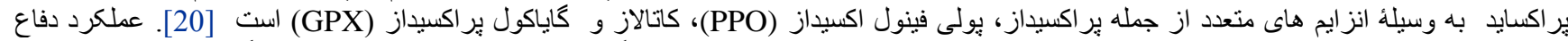

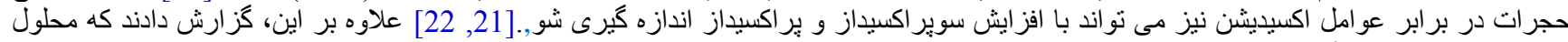

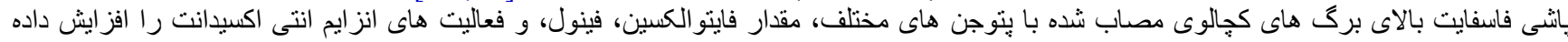

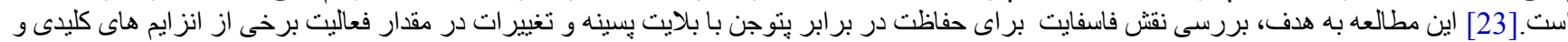

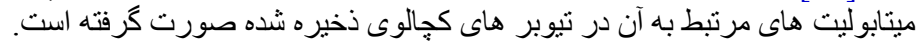

2 1.2

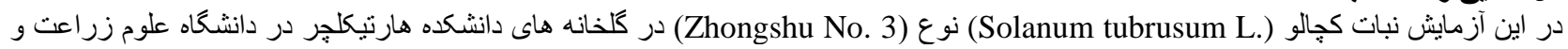

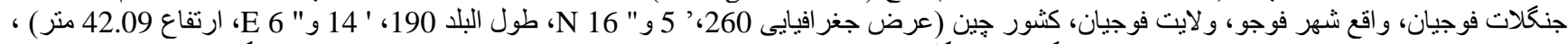

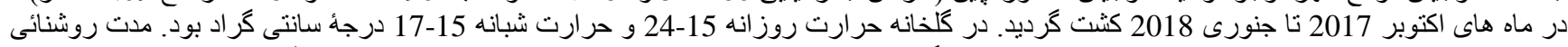

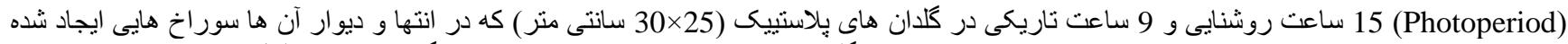

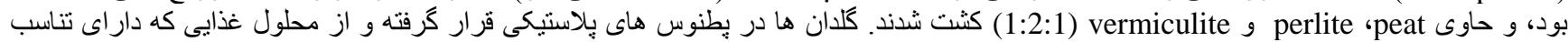
مختلف (نايتروجن فاسفورس و ويتاشيم) بر اي تغذيه نباتات از قسمت زيرين استفاده شد.

2.2

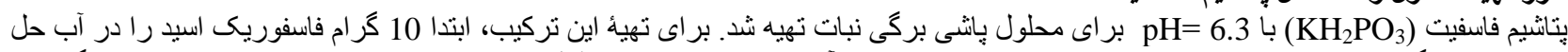

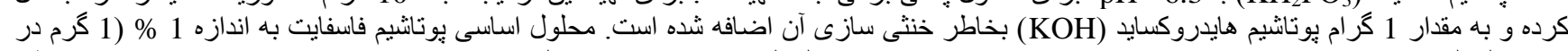

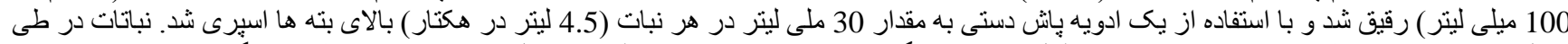

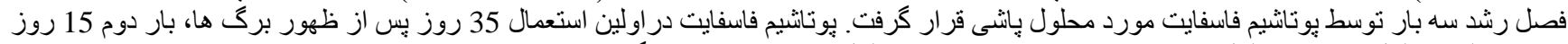

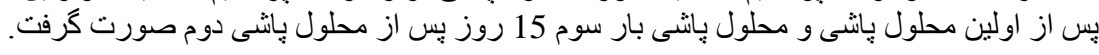

3.2

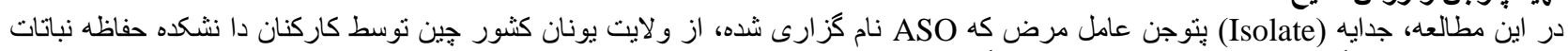

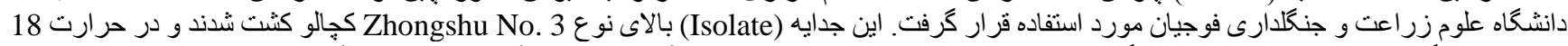

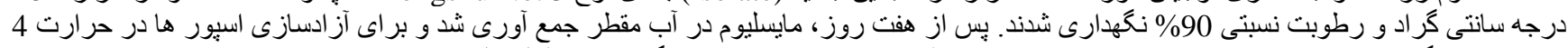

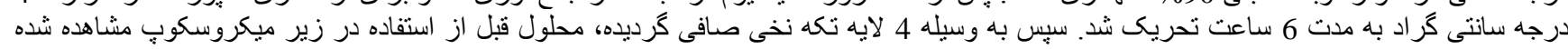

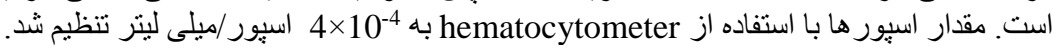

4.2

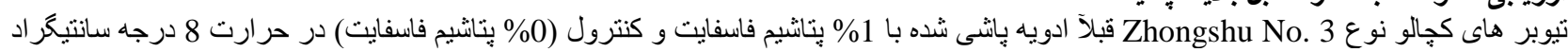

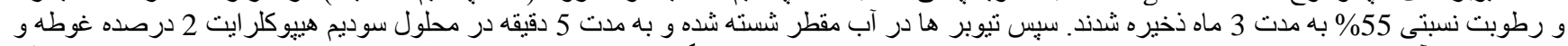

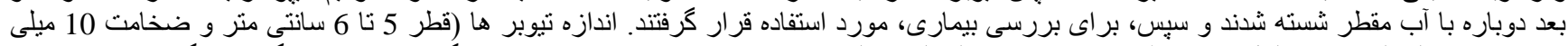

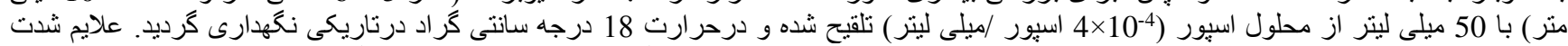

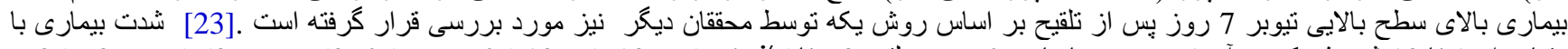

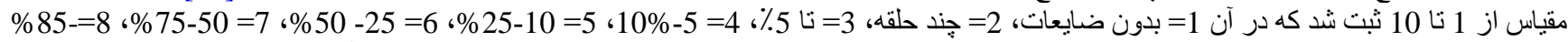

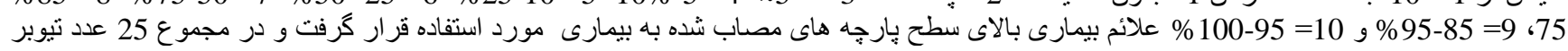

درتيمار در سه تكرار استفاده شد.

5.2

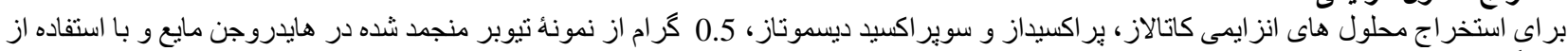

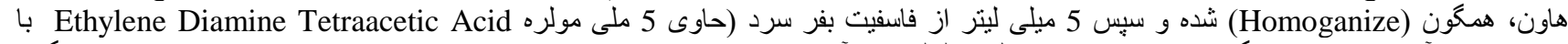

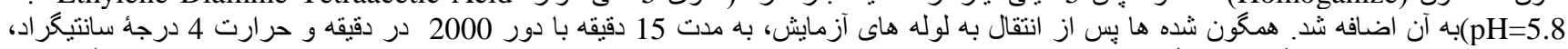

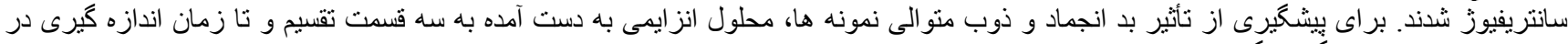

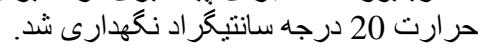

(SOD) 6.2

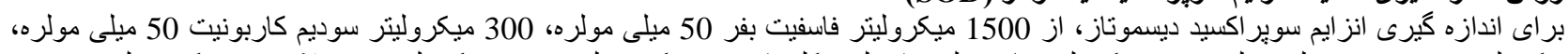

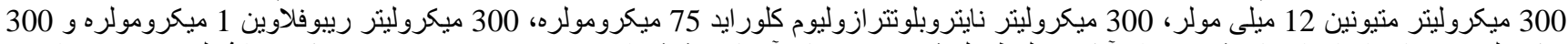

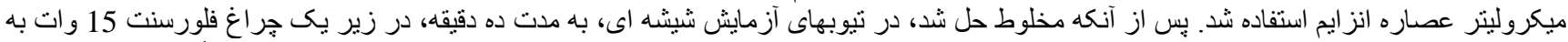

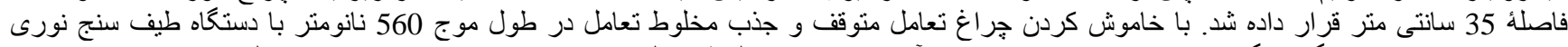

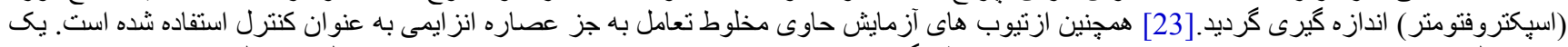

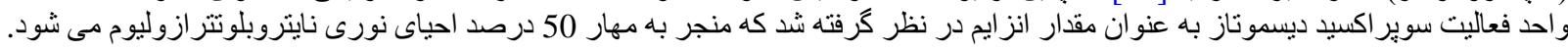

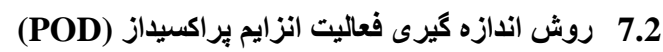

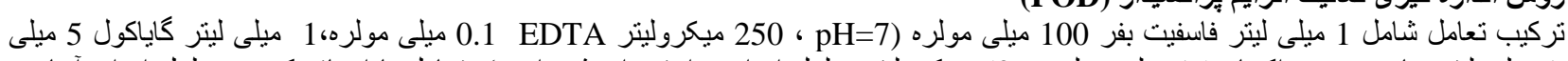

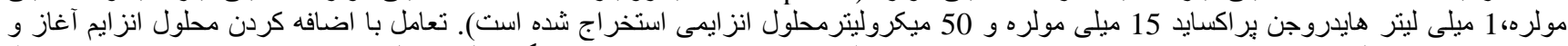

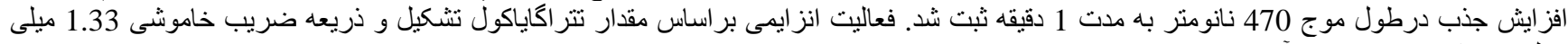
مول فى سانتى مثر به دست درج آمد [20] 
(CAT) 8.2

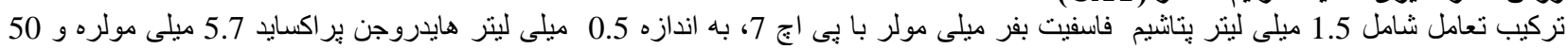

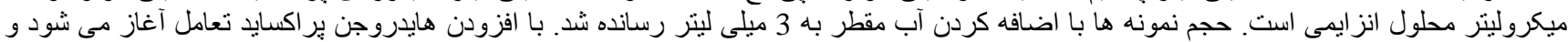

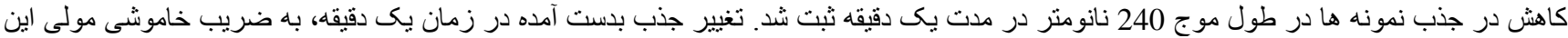

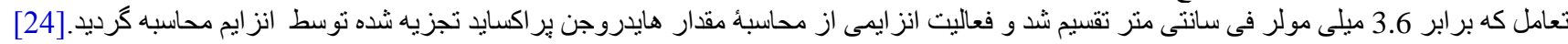

(Phytoalexin) 9.2

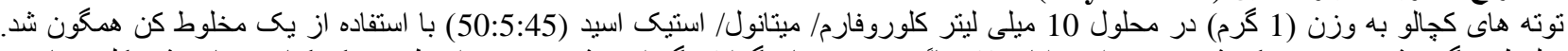

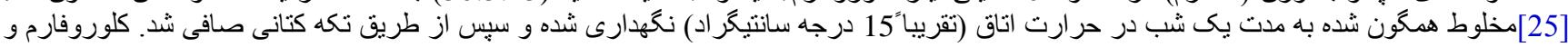

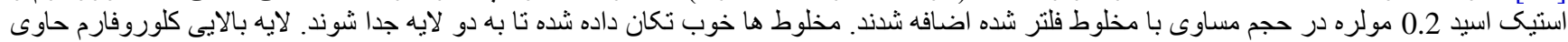

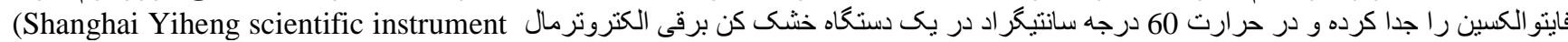
(H2SO (H) به محلول اضافه كرديده، و مخلوط

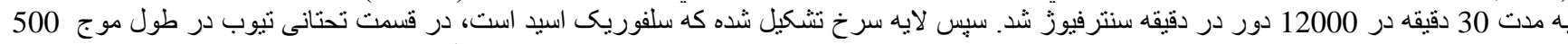

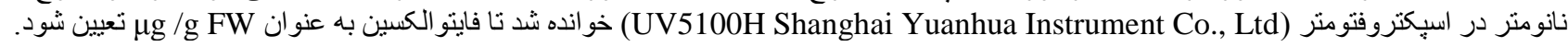

(Phenolic) 10.2

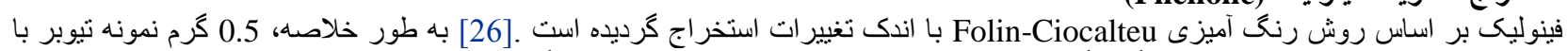

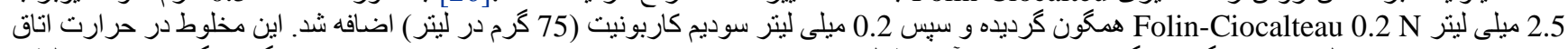

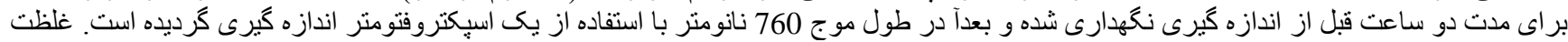

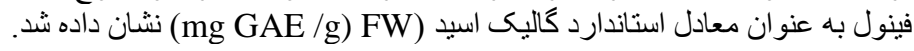

11.2

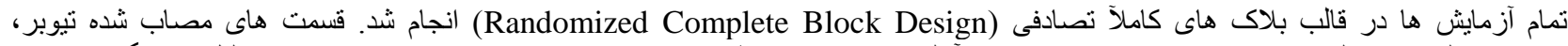

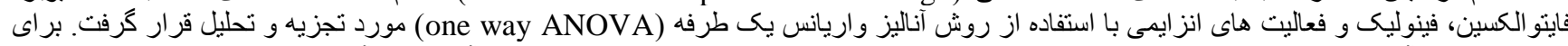

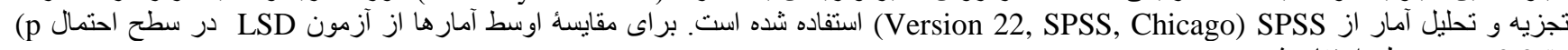
(0.05)

1.3

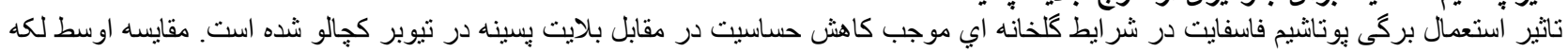

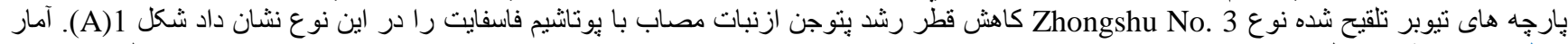

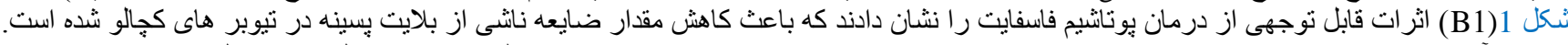

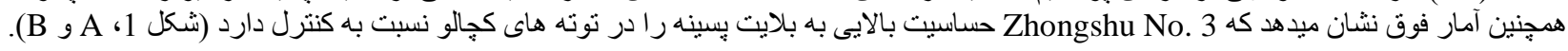

$\mathbf{A}$

(شاهد)كنترول

فاسفيت بتاشيح

B

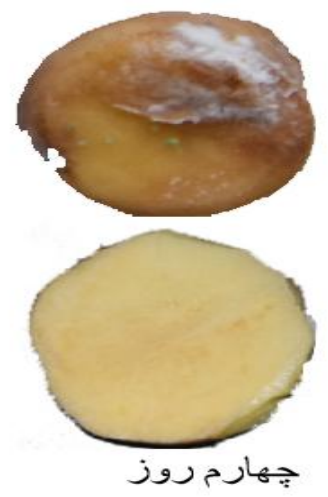

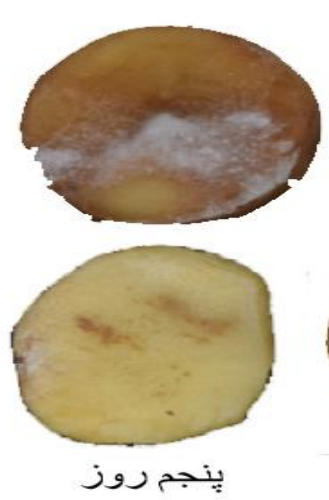
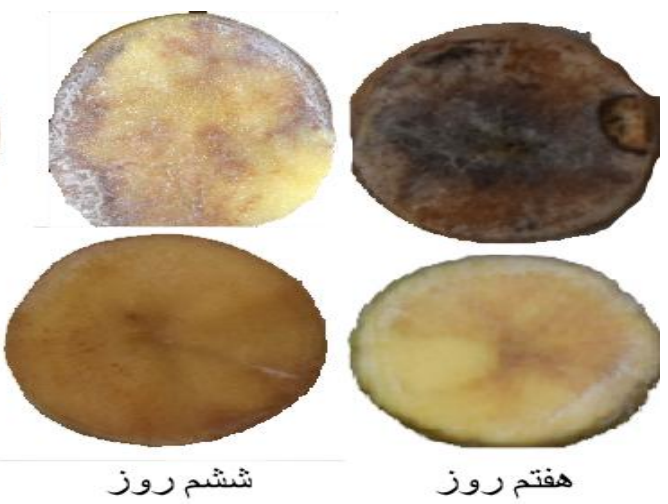

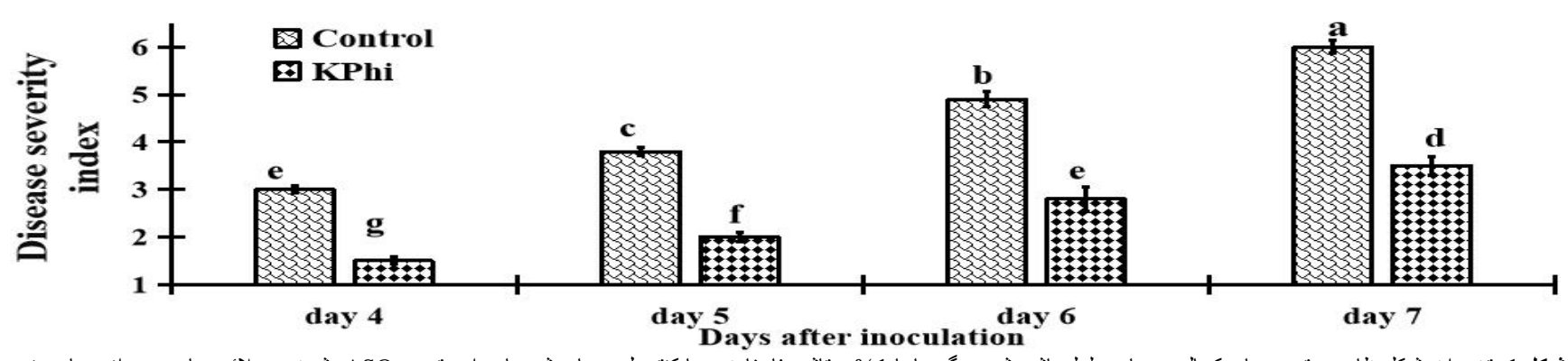

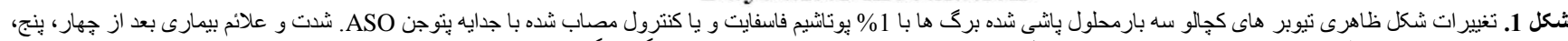

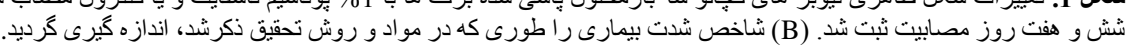

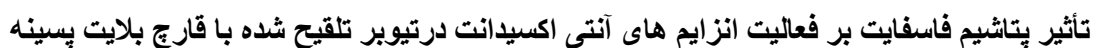

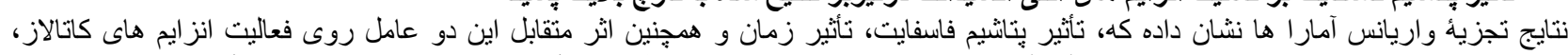

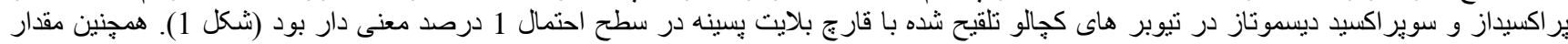




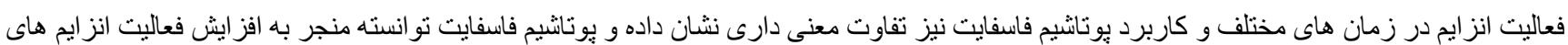

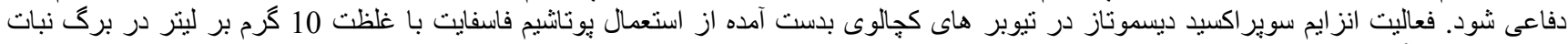

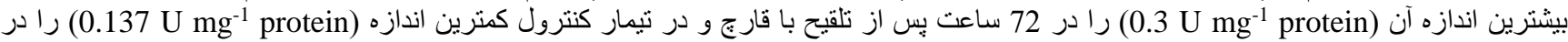

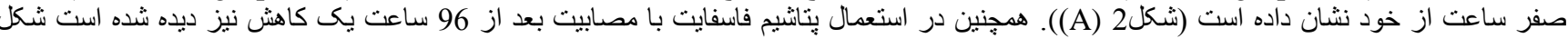

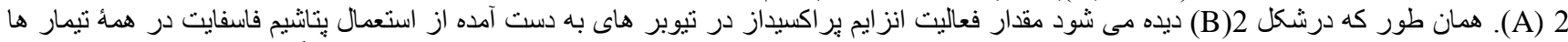

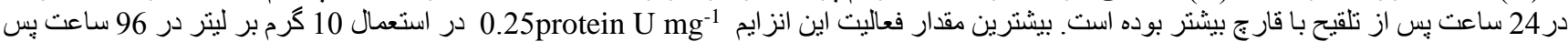

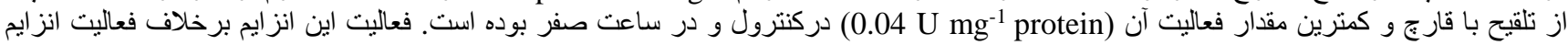

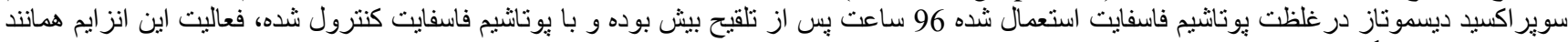

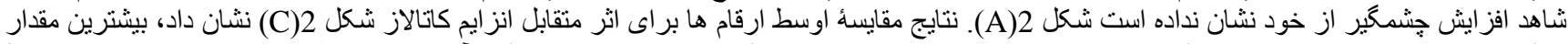

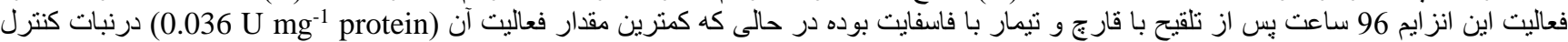

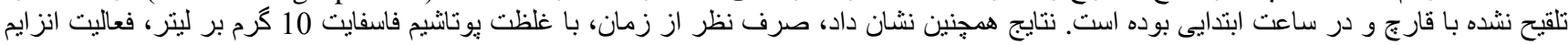

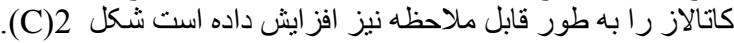

A

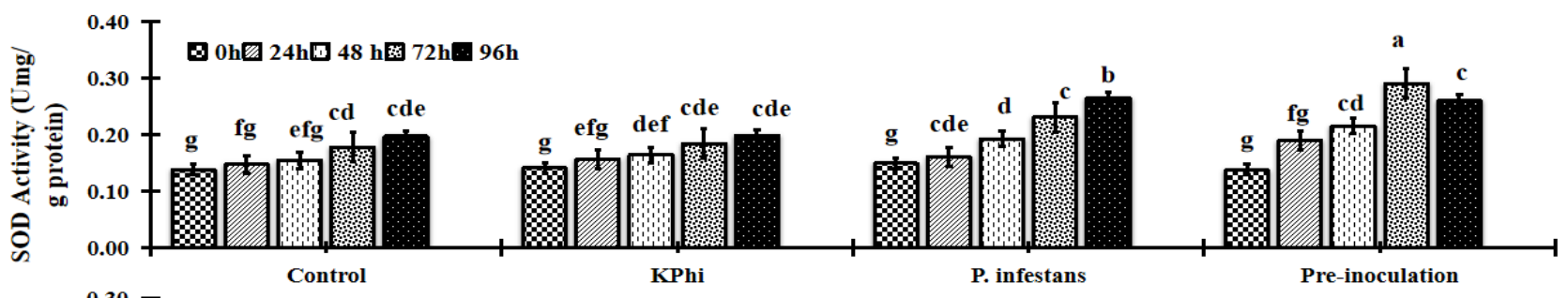

B

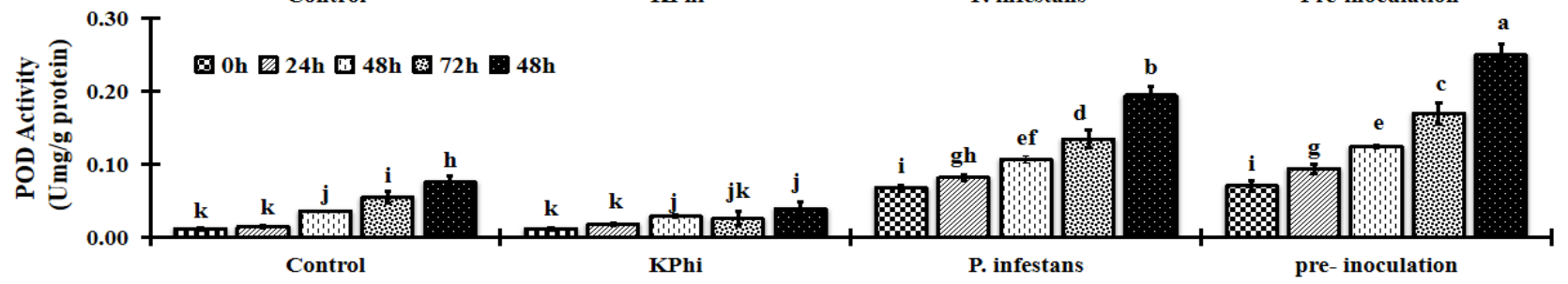

C

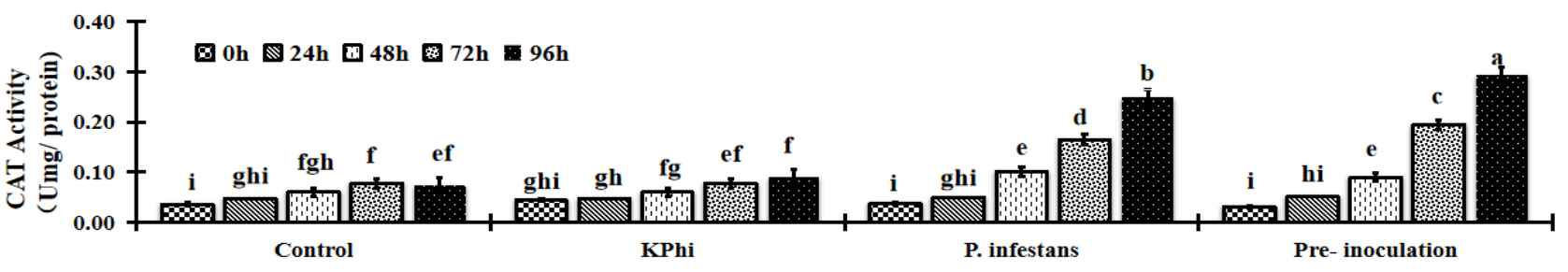

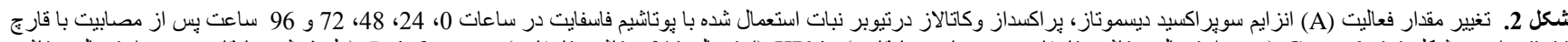

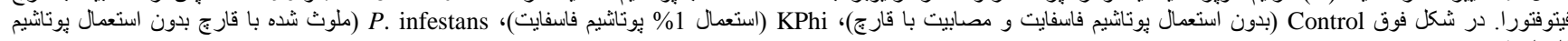

فاسفايت).

3.3

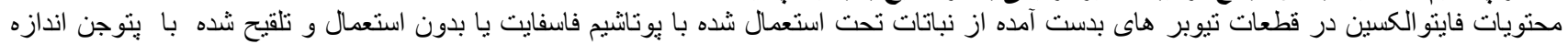

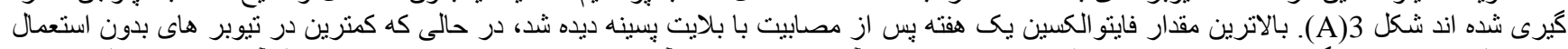

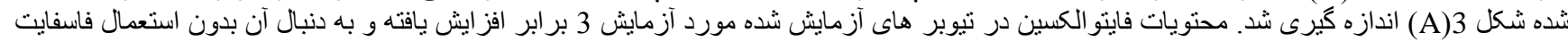

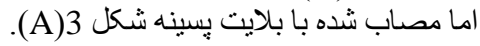

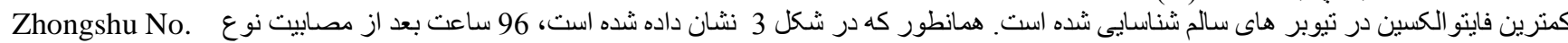

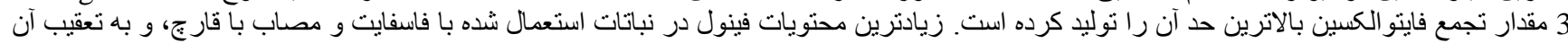

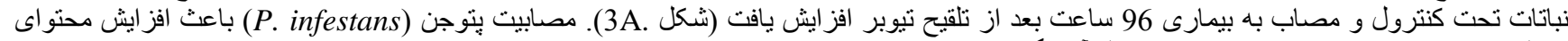

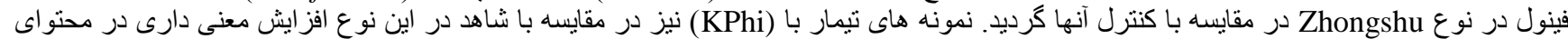

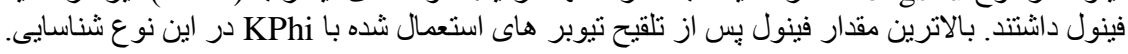

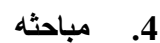

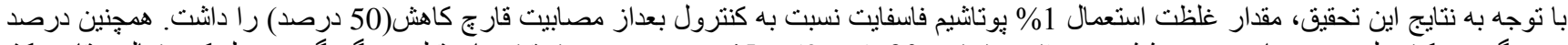

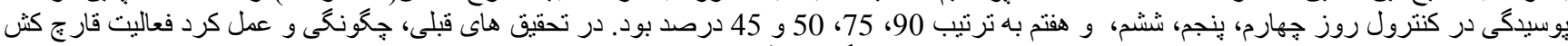

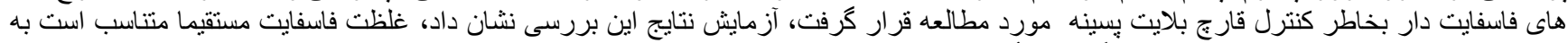

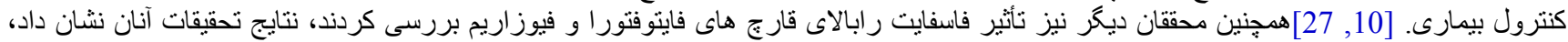

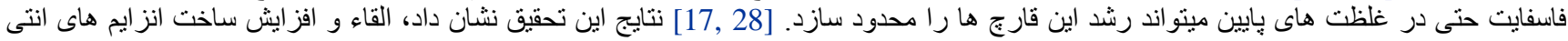

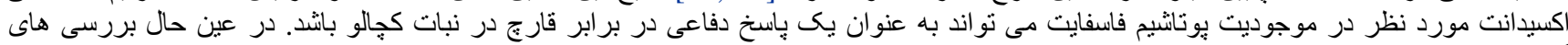

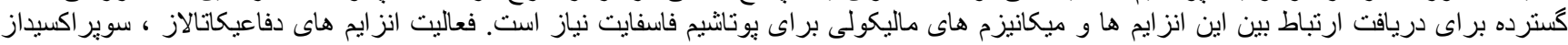

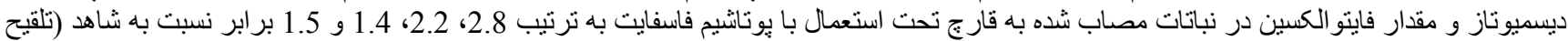
نشده با قارع) افزايش يافته است. اين دائ درحالى است كه مقدار فينول، 
A

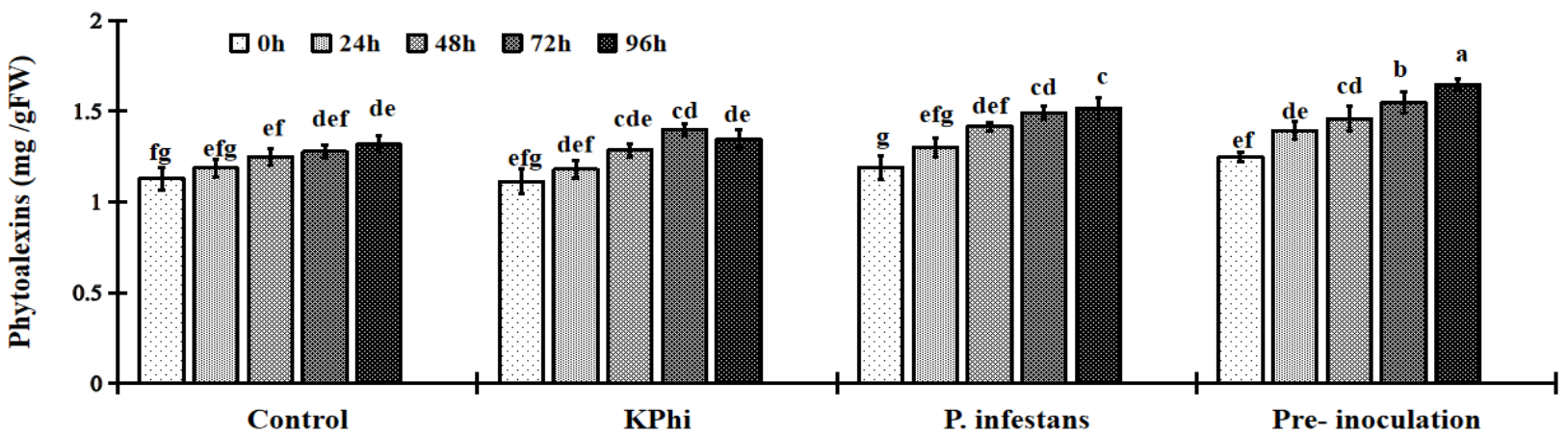

$B$
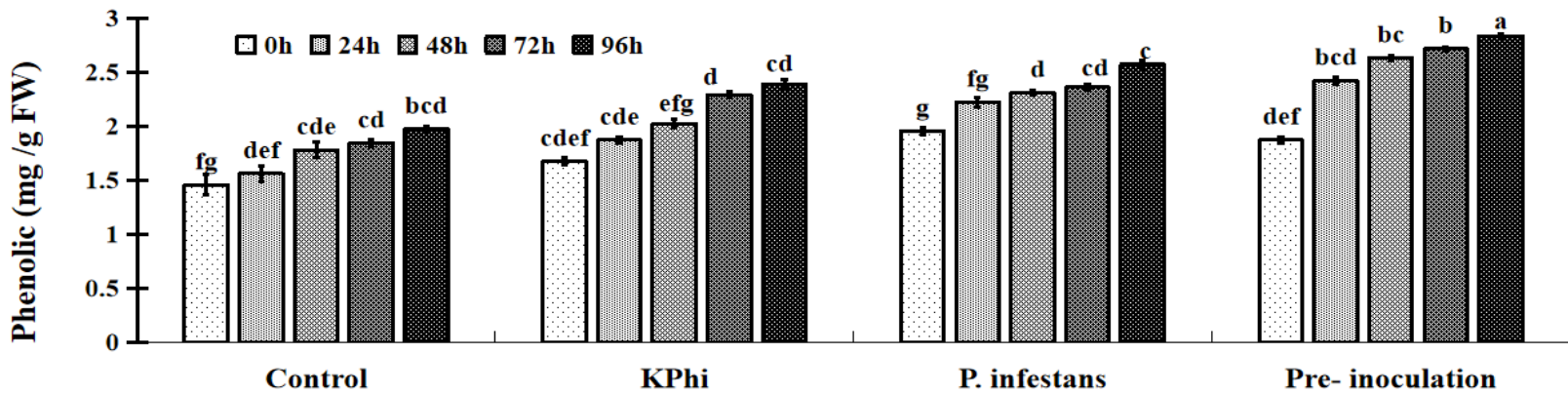

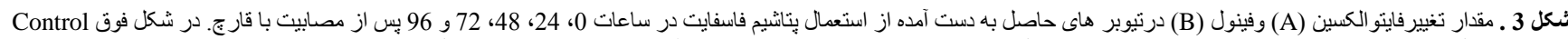

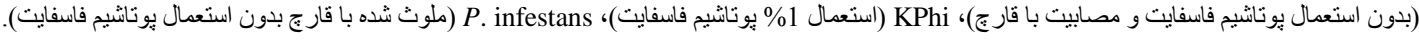

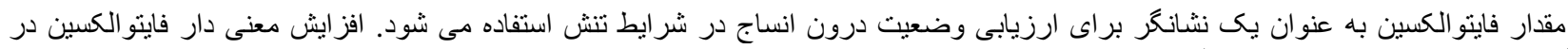

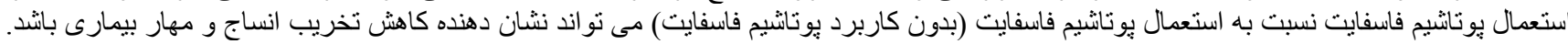

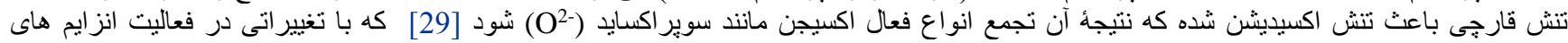

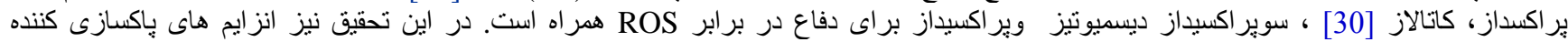

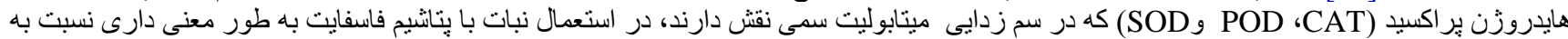

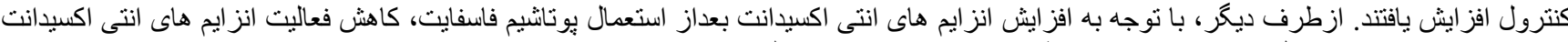

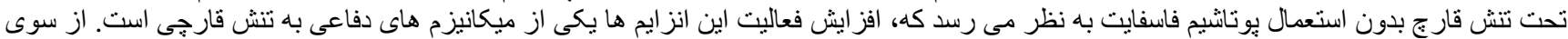

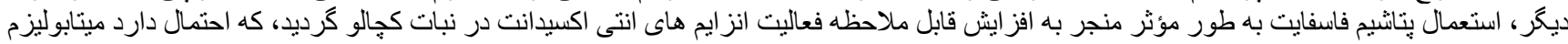

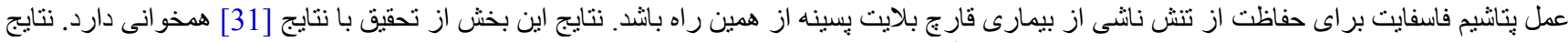

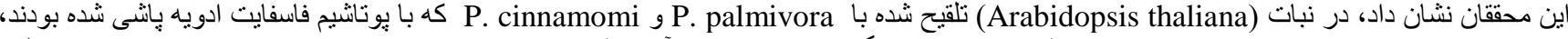

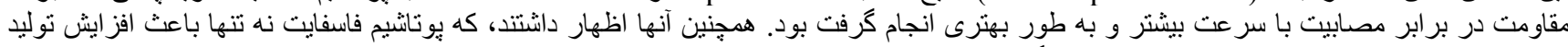

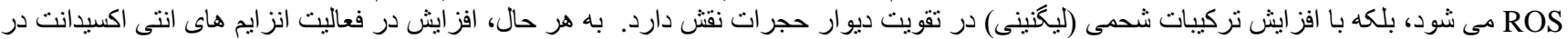

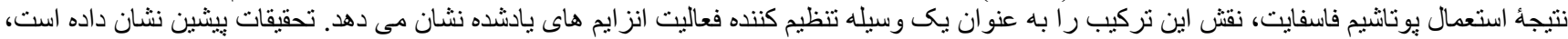

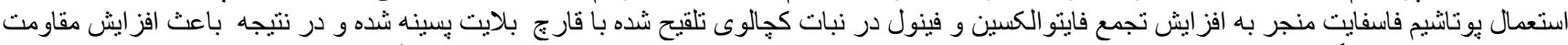

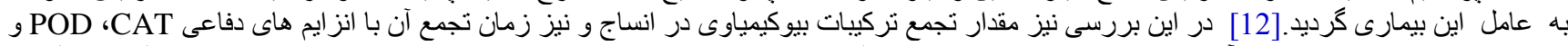

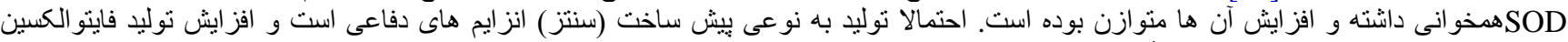

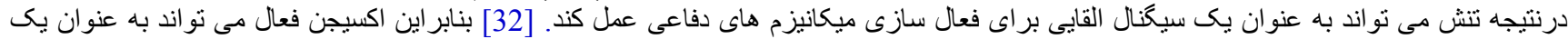

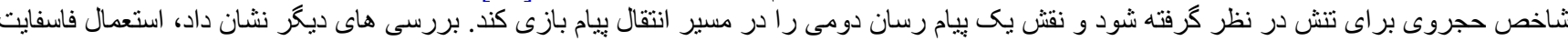

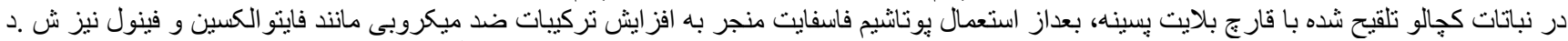

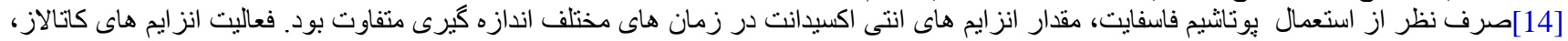

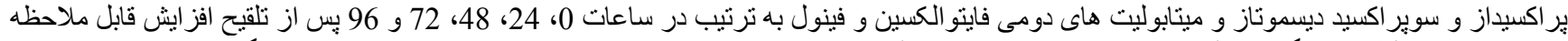

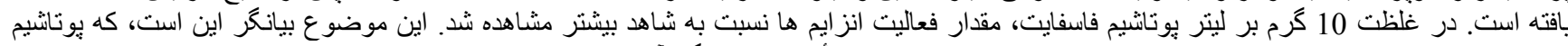

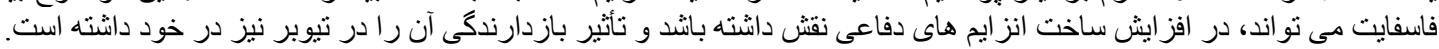

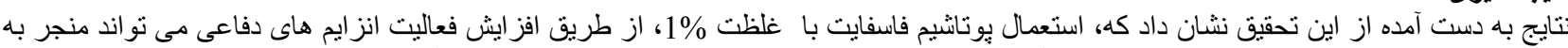
5

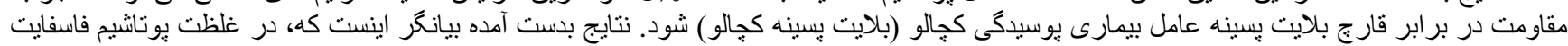

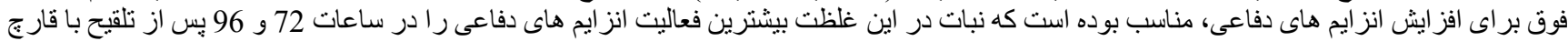

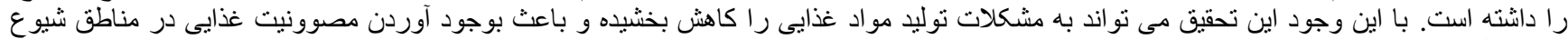

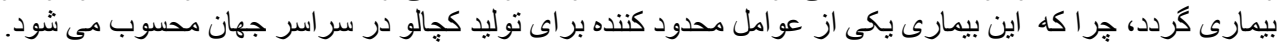

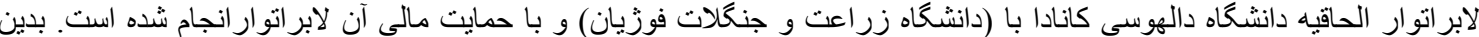
وسيله از مديرو كاركنان اين لابر اتوار ، تشكر و قدردانى ميكردد. 


\section{References}

[1] G. W. Norton and S. M. Swinton, "Precision agriculture: Global prospects and environmental implications, Tomorrow's Agriculture: Incentives, Institutions," in Infrastructure and Innovations-Proceedings of the Twenty-fouth International Conference of Agricultural Economists, 2018, p. 269.

[2] M. C. Lobato, G. R. Daleo, A. B. Andreu, and F. P. Olivieri, "Cell Wall Reinforcement in the Potato Tuber Periderm After Crop Treatment with Potassium Phosphite," Potato Research, pp. 1-11, 2017.

[3] T. S. Lim, R .D. Borza, R. H. Peters, K. I. Coffin, D. M. Al-Mughrabi, G. Pinto, and Wang-Pruski, "Proteomics analysis suggests broad functional changes in potato leaves triggered by phosphites and a complex indirect mode of action against phytophthora infestans," Journal of Proteomics, vol. 93, pp. 207-223, 2013.

[4] Y. Xi, X. Han, Z. Zhang, J. Joshi, T. Borza, M. M. Aqa, B. Zhang, H. Yuan, and G. Wang-Pruski, "Exogenous phosphite application alleviates the adverse effects of heat stress and improves thermotolerance of potato (Solanum tuberosum L.) seedlings," Ecotoxicology and Environmental Safety, vol. 190, p. 110048, 2020.Available at: https://doi.org/10.1016/j.ecoenv.2019.110048 .

[5] O. Silva, H. Santos, M. Dalla Pria, and L. May-De Mio, "Potassium phosphite for control of downy mildew of soybean," Crop Protection, vol. 30, pp. 598-604, 2011.Available at: https://doi.org/10.1016/j.cropro.2011.02.015 .

[6] A. E. McDonald, B. R. Grant, and W. C. Plaxton, "Phosphite (phosphorous acid): Its relevance in the environment and agriculture and influence on plant phosphate starvation response," Journal of Plant Nutrition, vol. 24, pp. 1505-1519, 2001.Available at: https://doi.org/10.1081/pln-100106017 .

[7] R. Daniel and D. Guest, "Defence responses induced by potassium phosphonate in phytophthora palmivora-challenged Arabidopsis thaliana," Physiological and Molecular Plant Pathology, vol. 67, p. 194 to 201, 2005.Available at: https://doi.org/10.1016/j.pmpp.2006.01.003 .

[8] H. T. B. Thao and T. Yamakawa, "Phosphite (phosphorous acid): Fungicide, fertilizer or bio-stimulator?," Soil Science and Plant Nutrition, vol. 55, pp. 228-234, 2009.Available at: https://doi.org/10.1111/j.1747-0765.2009.00365.x .

[9] T. Borza, R. Peters, Y. Wu, A. Schofield, J. Rand, Z. Ganga, K. Al-Mughrabi, R. Coffin, and G. Wang-Pruski, "Phosphite uptake and distribution in potato tubers following foliar and postharvest applications of phosphite-based fungicides for late blight control," Annals of Applied Biology, vol. 170, pp. 127-139, 2017.Available at: https://doi.org/10.1111/aab.12322

[10] G. Wang-Pruski, R. H. Coffin, R. D. Peters, K. I. Al-Mughrabi, H. W. Platt, D. Pinto, S. Veenhuis-MacNeill, W. Hardy, S. Lim, and T. Astatkie, "Phosphorous acid for late blight suppression in potato leaves," Am. J. Plant Sci. Biotechnol, vol. 4, 2010 .

[11] T. Borza, A. Schofield, G. Sakthivel, J. Bergese, X. Gao, J. Rand, and G. J. C. p. Wang-Pruski, " Ion chromatography analysis of phosphite uptake and translocation by potato plants: dose-dependent uptake and inhibition of Phytophthora infestans development," vol. 56, pp. 74-81, 2014.Available at: https://doi.org/10.1016/j.cropro.2013.10.024 .

[12] M. F. Machinandiarena, M. C. Lobato, M. L. Feldman, G. R. Daleo, and A. B. Andreu, "Potassium phosphite primes defense responses in potato against phytophthora infestans," Journal of Plant Physiology, vol. 169, pp. 1417-1424, 2012.Available at: https://doi.org/10.1016/j.jplph.2012.05.005 .

[13] L. Wiesel, A. C. Newton, I. Elliott, D. Booty, E. M. Gilroy, I. Birch, and P. R. Hein, "Molecular effects of resistance elicitors from biological origin and their potential for crop protection," Frontiers in Plant Science vol. 5, p. 655, 2014.Available at: https://doi.org/10.3389/fpls.2014.00655 .

[14] M. Chandrasekaran, S. T. Belachew, E. Yoon, and S. C. Chun, "Expression of $\beta$-1, 3-glucanase (GLU) and phenylalanine ammonia-lyase (PAL) genes and their enzymes in tomato plants induced after treatment with Bacillus subtilis CBR05 against Xanthomonas campestris pv. vesicatoria," Journal of General Plant Pathology, vol. 83, pp. 7-13, 2017.Available at: https://doi.org/10.1007/s10327-016-0692-5 .

[15] H. K. Kim, Y. H. Choi, and R. Verpoorte, "NMR-based metabolomic analysis of plants," Nature Protocols, vol. 5, p. 536, 2010.Available at: https://doi.org/10.1038/nprot.2009.237 .

[16] J. Kuć, "Concepts and direction of induced systemic resistance in plants and its application," European Journal of Plant Pathology, vol. 107, pp. 7-12, 2001.Available at: https://doi.org/10.1023/A:1008718824105 .

[17] R. J. Dalio, F. Fleischmann, M. Humez, and W. Osswald, "Phosphite protects fagus sylvatica seedlings towards phytophthora plurivora via local toxicity, priming and facilitation of pathogen recognition," PloS One, vol. 9, p. e87860.2014 ,Available at: https://doi.org/10.1371/journal.pone.0087860.

[18] M. Ramezani, F. Rahmani, and A. Dehestani, "Study of physio-biochemical responses elicited by potassium phosphite in downy mildew-infected cucumber plants," Archives of Phytopathology and Plant Protection, vol. 50, pp. 540-554, 2017.Available at: https://doi.org/10.1080/03235408.2017.1341140 .

[19] B. Seckin, I. Turkan, A. H. Sekmen, and C. Ozfidan, "The role of antioxidant defense systems at differential salt tolerance of Hordeum marinum Huds.(sea barleygrass) and Hordeum vulgare L.(cultivated barley)," Environmental and Experimental Botany, vol. 69, pp. 76-85, 2010.Available at: https://doi.org/10.1016/j.envexpbot.2010.02.013 .

[20] B. Debnath, M. Irshad, S. Mitra, M. Li, H. M. Rizwan, S. Liu, T. Pan, and D. Qiu, "Acid rain deposition modulates photosynthesis, enzymatic and non-enzymatic antioxidant activities in tomato," International Journal of Environmental Research, vol. 12, pp. 203-214, 2018.Available at: https://doi.org/10.1007/s41742-018-0084-0 .

[21] B. Debnath, M. Hussain, M. Li, X. Lu, Y. Sun, and D. Qiu, "Exogenous melatonin improves fruit quality features, health promoting antioxidant compounds and yield traits in tomato fruits under acid rain stress," Molecules, vol. 23 ,p. 1868 , 2018.Available at: https://doi.org/10.3390/molecules23081868 .

[22] P. Majer, G. Czégény, G. Sándor, P. J. Dix, and É. Hideg, "Antioxidant defence in UV-irradiated tobacco leaves is centred on hydrogen-peroxide neutralization," Plant Physiology and Biochemistry, vol. 82, pp. 239-243, 2014.Available at: https://doi.org/10.1016/j.plaphy.2014.06.011 .

[23] M. Mohammadi, Z. Zhang, Y. Xi, H. Han, F. Lan, B. Zhang, and G. Wang-Pruski, "Effects of potassium phosphite on biochemical contents and enzymatic activities of chinese potatoes inoculated by phytophthora infestans," Applied Ecology and Environmental Research, vol. 17, pp. 4499-4514, 2019.Available at: https://doi.org/10.15666/aeer/1702_44994514 .

[24] I. M. Ahmed, F. Cao, M. Zhang, X. Chen, G. Zhang, and F. Wu, "Difference in yield and physiological features in response to drought and salinity combined stress during anthesis in Tibetan wild and cultivated barleys," PloS One, vol. 8, p. e77869, 2013.Available at: https://doi.org/10.1371/journal.pone.0077869 . 
[25] M. Lobato, F. Olivieri, E. G. Altamiranda, E. Wolski, G. Daleo, D. Caldiz, and A. Andreu, "Phosphite compounds reduce disease severity in potato seed tubers and foliage," European Journal of Plant Pathology, vol. 122, pp. 349-358, 2008.Available at: https://doi.org/10.1007/s10658-008-9299-9 .

[26] M. Škerget, P. Kotnik, M. Hadolin, A. R. Hraš, M. Simonič, and Ž. Knez, "Phenols, proanthocyanidins, flavones and flavonols in some plant materials and their antioxidant activities," Food Chemistry, vol. 89, pp. 191-198, 2005.Available at: https://doi.org/10.1016/j.foodchem.2004.02.025 .

[27] E. Liljeroth, A. Lankinen, L. Wiik, D. D. Burra, E. Alexandersson, and E. Andreasson, "Potassium phosphite combined with reduced doses of fungicides provides efficient protection against potato late blight in large-scale field trials," Crop Protection, vol. 86, pp. 42-55, 2016.Available at: https://doi.org/10.1016/j.cropro.2016.04.003 .

[28] A. F. L. Cerqueira, Effects of phosphite in Pinus radiata-Fusarium circinatum interaction: Universidade de Aveiro, 2016.

[29] L. Wu, X. Gao, F. Xia, J. Joshi, T. Borza, and G. Wang-Pruski, "Biostimulant and fungicidal effects of phosphite assessed by GC-TOF-MS analysis of potato leaf metabolome," Physiological and Molecular Plant Pathology, vol. 106, pp. 49-56, 2019.Available at: https://doi.org/10.1016/j.pmpp.2018.12.001 .

[30] M. Mofidnakhaei, V. Abdossi, A. Dehestani, H. Pirdashti, and V. Babaeizad, "Potassium phosphite affects growth, antioxidant enzymes activity and alleviates disease damage in cucumber plants inoculated with Pythium ultimum," Archives of Phytopathology and Plant Protection, vol. 49, pp. 207-221, 2016.Available at: https://doi.org/10.1080/03235408.2016.1180924 .

[31] D. B. Lobell, W. Schlenker, and J. Costa-Roberts, "Climate trends and global crop production since 1980," Science, vol. 333, pp. 616-20, 2011.Available at: https://doi.org/10.1126/science.1204531 .

[32] R. Hammerschmidt, "Phytoalexins: What have we learned after 60 years?," Annual Review of Phytopathology, vol. 37, pp. 285-306, 1999.Available at: https://doi.org/10.1146/annurev.phyto.37.1.285 . 